\title{
Fire-prone areas delineated from a combination of the Nesterov Fire-risk Rating Index with multispectral satellite data
}

\author{
Milan Onderka $\cdot$ Igor Melicherčik
}

Received: 21 April 2009 /Accepted: 19 November 2009/Published online: 9 December 2009

(C) Società Italiana di Fotogrammetria e Topografia (SIFET) 2009

\begin{abstract}
Traditional fire-risk rating indices are founded on statistical relations between pre-event meteorological conditions and the number of fire outbreaks observed in a forested area. However, traditional weather-based indices cannot render information on the spatial distribution of firesusceptible sites over extensive forested areas because their only inputs are meteorological observations made at sparsely distributed weather stations. Therefore, only an area-averaged value of the risk of fire can be obtained from these indices. This disadvantage can be relieved by using remote sensing data from polar-orbiting satellites scanning the Earth's surface in the visible and thermal spectral regions. This paper presents a synthesis of the Nesterov Fire-rating Index, an index extensively used in Central and Eastern Europe- and how this was merged with the Temperature-Vegetation Dryness Index (TVDI) derived from multispectral images scanned by Landsat ETM+. This paper demonstrates the methodology of how TVDI was merged with the Nesterov Index to give a map of the spatial patterns of fire-prone sites. The test region is located in a pine-forested (dominated by Pinus sylvestris) area in western Slovakia. Our investigation suggests that coupling TVDI with the Nesterov Index, or any other weather-based fire-rating index, can become an effective tool for delineating areas prone to fire outbreaks
\end{abstract}

\footnotetext{
M. Onderka $(\square)$

Institute of Hydrology, SAS,

Račianska 75,

83201 Bratislava, Slovakia

e-mail: onderka@uh.savba.sk

I. Melicherčik

Faculty of Mathematics, Physics and Informatics,

Comenius University,

Mlynská dolina,

84248 Bratislava, Slovakia
}

even in regions with insufficient coverage of weather stations.

Keywords Wildfire - Forest fire - TVDI · Landsat ETM+ . Nesterov Index

\section{Introduction}

The likelihood that a fire will break out in a forest is traditionally assessed by deploying empirical indices using basic meteorological data. There are several indices in use worldwide, e.g., the Nesterov Index (in Russia), Angstom Index (Northern Europe), Baumgartner Index (Germany), and the Canadian Fire Weather Index. The fundamental feature of weather-based indices is that they are based on simplified statistical relationships between the reported number of fire events and several weather data characterizing the pre-event weather conditions. However, weather-based indices can provide only an area-averaged risk of fire, as their only input (weather data) is acquired from point measurements taken at representative weather stations. The climate, topography, and the type of vegetation are recognized as additional factors modulating the fire potential and the rate of spread (Škvarenina et al. 2004). Microclimatic conditions, i.e., temperature of the forest floor along with other favorable conditions (high air temperature, low air humidity, direct solar radiation, and wind) are the main factors facilitating ignition and rapid spread of fire in forests (Tanskanen et al. 2005; Bowyer and Danson 2004). There is evidence that fuel moisture content and surface soil moisture are both controlled by the amount of water vapor in the air, rainfall interception, and direct solar heating. Tesař et al. (2006) showed that the density of a forest overstory directly affects the surface radiation balance and surface temperature of the forest floor. 
There is evidence that surface moisture and temperature in large gaps are both controlled primarily by direct solar heating and evaporation. Enhanced ventilation in gaps and thin forests further contributes to drying of litter in addition to the direct solar heating and evaporation. Forest microclimate is controlled also by the species composition and the vertical structure of canopy by affecting the intensity of light penetrating the canopy and vertical fluxes of heat and vapor. The vertical structure of forest has been recently modeled by means of LIDAR as an innovative approach (Richardson et al. 2009) with promising implication to fire-risk modeling. Generally, Scots pine (Pinus sylvestris) creates stand structures allowing wind and sun radiation to penetrate tree canopy making this specie more prone to fire than, e.g., Norway spruce (Picea abies), (Tanskanen et al. 2005; Tanskanen and Venalainen 2008).

Generally, two types of flammable fuel are considered in fire-risk modeling: dead fuel and live fuel (i.e., green vital leaves, branches, etc.). While live fuel plays an essential role in crown fire development and the rate of its spread (Garcia-Martin et al. 2008; Peterson et al. 2008; Chuvieco et al. 2004), dead fuel (i.e., dry branches, shrubs, and litter) serve as the primary fuel material for human-caused fire ignitions. As Bowyer and Danson (2004) emphasize, LFM varies both in space and time and is driven primarily by the available soil moisture, the type of the underlying soil, and most importantly, the microclimate within the forest canopy. Dead forest fuel is moistened by precipitation that falls on the forest floor after passing through the canopy ("throughfall"). However, it has to be noted that the temporal variability of precipitation is a matter of season, but high variability may occur within a single storm as well. Distribution of rainfall varies with storm type, rainfall intensity, duration, and time of year (Bedient et al. 2008). Since rainfall is principal source of moisture for forest fuel, spatial variability of precipitation plays a significant role in the spatial distribution of fire-prone sites.

The advent of remote sensing made it possible to obtain spatial characteristics of forest biomass and surface moisture conditions. Vegetation indices (VIs) derived from remotely sensed data have been used to assess the state of vegetation by diagnosing its spectral features in the visible and the nearmedium infrared regions of the electromagnetic spectrum. Estimation of soil moisture, which is an important driver of water vapor fluxes and heat transport in forests, has been shown to be retrievable from VIs (Wang et al. 2007). In firerelated studies, VIs derived from satellite data have been used to map extensive areas of dry vegetation. A strong linkage was found between crown biomass derived from Landsat images and burnt severity (Garcia-Martin et al. 2008). With decreasing content of water in fuel, spectral characteristics of the biomass change as a response to water deficiency, since leaf reflectance is controlled mainly by water content, pigments (such as the chlorophyll-a and chlorophyll-b), and dry matter. VIs are sensitive to changes in vegetation chlorophyll absorption and leaf area index (LAI), which co-occur with changes in water stress (Hardy and Burgan 1999). Because soil moisture is partially driven by vegetation cover, Normalized Differential Vegetation Index (NDVI; along with other vegetation indices) are useful for assessing the spatial variability of soil and fuel moisture (Danson and Bowyer 2004). Nemani et al. (1993) and Park et al. (2004) demonstrated the existence of a close relationship between soil moisture and NDVI. Wang et al. (2007) investigated the time lag of NDVI behind decreasing soil water content. For humid regions, this time lag may be more than 10 days, while in semi-arid regions NDVI may start to respond to water stress earlier $(<5$ days). Significant correlation was found between NDVI and shallow soil moisture at a depth of $50 \mathrm{~mm}$ (Wang et al. 2007). Considering the dead fuel and shallow soil horizons are closely interlinked in their water-energy balance, the reported correlation between NDVI and soil moisture in the most upper horizon of the soil may bear some information on the state of fuels. Hernandez-Leal et al. (2008) used the NDVI scanned by AVHRR-NOAA and Terra-MODIS channels to track seasonal changes in the vegetation status in order to assess the risk of fire in Spain. Hernandez-Leal et al. (2008) worked toward developing a Dynamic Fire-Risk Index that would incorporate NDVI into a Static Fire Index, i.e., an index utilizing factors such as proximity to main roads, type of vegetation cover, insulation hours, slope, aspect, and elevation of the terrain. A similar fire-risk zonemapping methodology based on topographic and land-use maps was proposed by Xu et al. (2005).

The major limitation of relying merely on VIs in firerelated studies is that VIs may only slowly respond to water deficiency. Combining images taken in visible, near and mid-infrared, and thermal domains may capture changes in water deficiency faster than single VIs. The attempt to merge the thermal spectral region with visible and infrared spectral bands resulted in the temperature-vegetation space, also known as the Temperature-Vegetation Scatterplot. Sandholt et al. (2002) modified the Temperature-Vegetation Scatterplot to identify soil moisture conditions. The modification resulted in the Temperature-VegetationDifferential Index (TVDI), which is based on relating NDVI to surface temperature in a number of discrete steps.

The primary motivation of this paper is to show how the Nesterov Index can be used in synergy with remotely sensed data, particularly the Temperature-Vegetation Dryness Index-TVDI derived from four Landsat ETM+ images and is used to assign a spatial attribute to the Nesterov Index by differentiating between areas of high, medium, and low risk of fire. The partial goals are: (1) to calculate the Nesterov Index; (2) to process and analyze four Landsat ETM+ images, and to 


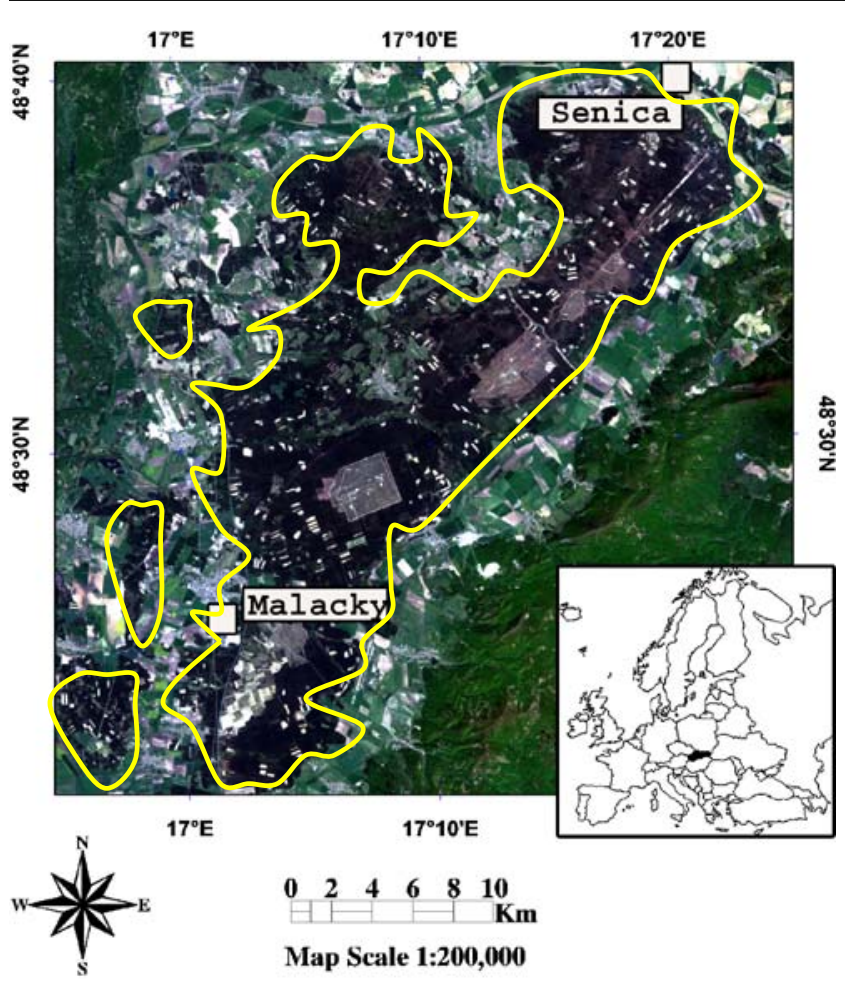

Fig. 1 Orthophoto-map of the investigated area. Locations of the weather stations at Malacky and Senica are indicated with rectangles (true-color composite RGB, created from Landsat imagery)

derive NDVI and surface brightness temperatures; and (3) to create maps of TVDI for the investigated area delineating sites with low, medium, and high risk of fire.

\section{Data and methodology}

Study site

The geographical setting of the region of interest is illustrated in Fig. 1. This area is located between $48^{\circ} 20^{\prime}$ $\mathrm{N} 17^{\circ} \mathrm{E}$ and $48^{\circ} 50^{\prime} \mathrm{N} 17^{\circ} 30^{\prime} \mathrm{E}$, northerly of the capital city of Bratislava, Slovakia. This region is dominated by vast areas ( 44,000 ha) of coniferous monocultures (Scots pine) growing on sandy soils ( $90 \%$ quartz). Some $35 \%$ of the forested area is formed by natural stands, and $65 \%$ occupy cultural forest plantations. The prevailing northwestern winds (annual average, $2.8 \mathrm{~m} / \mathrm{s}$ ), and low annual precipitation rates $(\sim 583 \mathrm{~mm})$ make these forests sensitive to fire events. As for the fire statistics in Slovakia, the year 2003 experienced 872 forest fire events with a total burnt area of 1,567 ha (European Commission, Report No. 5, 2004). The growing season in the investigated region lasts $\sim 250$ day, and the average air temperature during the peak growing season (June) is $19.6-20.2^{\circ} \mathrm{C}$.

Nesterov Index

In 1949, Nesterov (Shetinsky 1994) proposed a fire-risk rating index to be used in the former Soviet Union. This index establishes a range of discrete fire-risk levels. The Nesterov Index is calculated as follows:

$N I=\sum_{i=1}^{w}\left(T_{i}-T_{i}^{\mathrm{dew}}\right) T_{i}$

where NI denotes the Nesterov Index; $w$ is the number of days since the last rainfall exceeding $3 \mathrm{~mm} /$ day, $\mathrm{T}_{i}$ is the air temperature $\left({ }^{\circ} \mathrm{C}\right)$ on a given day; and $T_{i}^{\mathrm{dew}}$ is the dew point temperature $\left({ }^{\circ} \mathrm{C}\right.$ ). Once the daily rainfall (a cumulative value) exceeds $3 \mathrm{~mm}$ per day, the Nesterov Index has to be reset to "zero" (Shetinsky 1994; Škvarenina et al. 2004).

\section{Satellite image processing}

The satellite images used in this study were acquired on days with clear skies. Four Landsat ETM+ images were processed and analyzed. The dates of acquisition were: May 1, 2000; May 14, 2000; August 2, 2000; and August 24, 2002. More detail on the satellite images are provided in Table 1. Supervised classification was used to create maps of forest-only areas in the ENVI 4.3 software package. Landsat ETM+ scenes contain three visible bands (band 1 through band 3), three near and medium infrared bands (bands 4, 5, and 7) with a spatial resolution of $30 \mathrm{~m}$, and one thermal band with a resolution of $60 \mathrm{~m}$ (band 6), (Landsat Handbook, http://landsathandbook.gsfc.nasa.gov/ handbook.html). First, maps showing forest-only areas were extracted from raw satellite images by the supervised classification algorithm based on the maximum likelihood classification technique. Training pixels were collected from pre-selected regions of interest using all three visual bands (TM1-3), one near-infrared band (TM4), and the thermal band (TM6). The created classified image was then
Table 1 Earth-sun position characteristics on the acquisition dates and TVDI parameters ( $a$ and $b$ in Eq. 2)

\begin{tabular}{lccccc}
\hline Date & Azimuth & Sun elevation & $a$ values & $b$ values & $T_{\text {smin }}(\mathrm{K})$ \\
\hline May 1, 2001 & 148.2 & 52.8 & 305.83 & -25.7 & 299.2 \\
May 14, 2000 & 145.1 & 57.4 & 312.43 & -15.9 & 298.9 \\
August 2, 2000 & 143.6 & 54.0 & 309.48 & -14.2 & 302.0 \\
August 24, 2002 & 147.8 & 48.3 & 304.42 & -13.3 & 299.8 \\
\hline
\end{tabular}


Table 2 Nesterov Index (NI) calculated for the dates of satellite image acquisition for the two weather stations $\left(N I_{\text {Malacky }}\right.$ and $\left.N I_{\text {Senica }}\right)$

\begin{tabular}{lrrr}
\hline Date & $N I_{\text {Malacky }}$ & \multicolumn{1}{c}{$N I_{\text {Senica }}$} & \multicolumn{1}{c}{$* \overline{N I}$} \\
\hline May 14, 2000 & $5,156(\mathrm{H})$ & $1,224(\mathrm{M})$ & $3,190(\mathrm{M})$ \\
August 2, 2000 & $404(\mathrm{~L})$ & $351(\mathrm{~L})$ & $377.5(\mathrm{~L})$ \\
May 1, 2001 & $1,175(\mathrm{M})$ & $920(\mathrm{~L})$ & $1,047.5(\mathrm{M})$ \\
August 24, 2002 & $731(\mathrm{~L})$ & $1,252(\mathrm{M})$ & $991.5(\mathrm{~L})$ \\
\hline
\end{tabular}

The average index for the whole region (Malacky+Senica) is calculated as an arithmetic average $\overline{N I}$. The level of risk obtained from the Nesterov Index is indicated as $L$ low risk, $M$ medium risk, and $H$ high risk (see Fig. 3)

$* \overline{N I}=1 / 2\left(N I_{\text {Malacky }}+N I_{\text {Senica }}\right)$

used for binary masking to exclude areas other than coniferous forests from analysis.

Temperature/vegetation dryness index

To establish a spatially variable attribute of the Nesterov Index, we focused on the work of Sandholt et al. (2002). The concept of "Ts-Vegetation Space" was originally proposed to assess the soil moisture status of vegetation. In principle, the Ts-Vegetation Space represents a scatterplot of remotely sensed surface temperature and NDVI collected form a sample area with a broad range of moisture conditions. Goward et al. (1985) demonstrated a strong negative relationship between radiometric surface temperature and NDVI, which was explained by evaporative cooling of green live biomass. When surface conditions become drier, vegetated areas transpire less water, and, when a drought period prevails for a sufficiently long time, NDVI values decrease and surface temperature tends to increase due to hindered evaporative cooling. Figure 4 explains the underlying principles of the Temperature/Vegetation Dryness Index (TVDI) in detail. Calculation of the Temperature/Vegetation

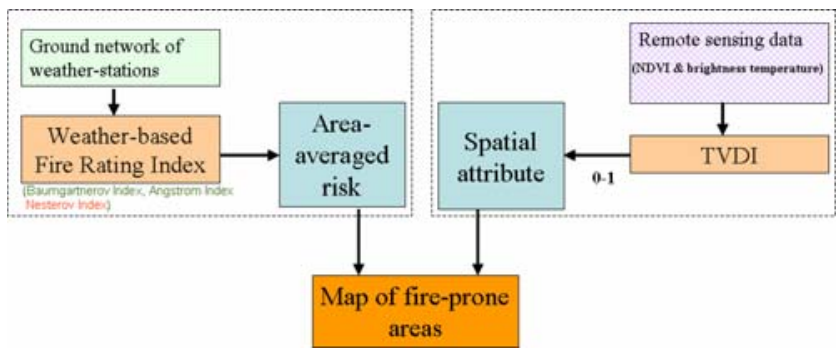

Fig. 2 Description of data sources used in creating a map of fireprone areas. The left-hand side box shows data acquired from ground observations (meteorological data) determining the risk of fire through the use of traditional weather-based indices (e.g., the Nesterov Index). The right-hand side box shows the remotely sensed information used to delineate dry and hot pixels within a satellite image using the Temperature-Vegetation Dryness Index (TVDI). Both data sources are merged into a map of fire-prone areas

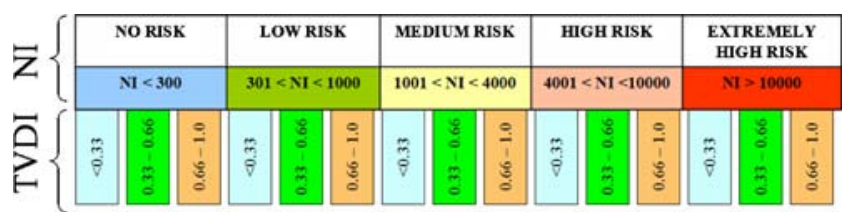

Fig. 3 Refinement of the discrete values of the Nesterov Index obtained from Eq. 1 by the spatially variable Temperature-Vegetation Index-TVDI (Eq. 2). Three intervals of TVDI are considered: $0-0.33$; $0.33-0.66$; and $0.66-1.0$

Dryness Index is based on the following equation (Sandholt et al. 2002):

$T V D I=\frac{T_{S}-T_{S_{-} \min }}{a+b \times N D V I-T_{S_{-} \min }}$

Where $T_{\mathrm{s}}$ is the surface temperature; $T_{\mathrm{s} \_ \text {min }}$ is the minimum surface temperature in the triangle necessary to define the "wet edge"; NDVI is the normalized differencial vegetation index; $a$ and $b$ are parameters derived from a linear fit to $T_{\mathrm{s} \_ \text {max }}=a+b N D V I$.

Note that the triangle in Fig. 4 is enclosed by two curves: the upper curve, called the "dry edge"; and the lower curve, called the "wet edge". The "wet edge" and "dry edge" in the triangle (Fig. 4) represent the TVDI boundary values. TVDI equals zero on the wet edge and unity (1) on the dry edge. All TVDI values between these two edges may therefore take values only in the range $0-1$.

\section{Retrieval of normalized differential vegetation index}

NDVI was calculated as the difference between NIR and Red spectral regions, normalized to the sum of the NIR and Red spectral band (Eq. 1):

$N D V I=\frac{\rho_{\mathrm{NIR}}-\rho_{\mathrm{Red}}}{\rho_{\mathrm{NIR}}+\rho_{\mathrm{Red}}}$



Fig. 4 Explanatory plot of the Temperature-Vegetation Dryness Index "TVDI" (adopted and partially modified from Sandholt et al. 2002) 
Fig. 5 TVDI scatterplots for the dates of image acquisition (May 1, 2001; May 14, 2000; August 2, 2000; and August 24, 2002). Legend: filled circle TVDI $\in(0-0.33)$; multiplication sign TVDI $\in(0.33-0.66)$;

$\Delta \mathrm{TVDI} \in(0.66-1.0)$


Where $\rho_{\mathrm{NIR}}$ is the spectral radiance detected in the nearinfrared band $(760-900 \mathrm{~nm}) ; \rho_{\text {Red }}$ is the spectral radiance detected in the red band $(630-690 \mathrm{~nm})$. The range of NDVI values can yield values only between -1 and 1 . As a rule of thumb, higher NDVI values characterize green and healthy biomass. For healthy coniferous forests (no or negligible water deficiency), NDVI may be within the range of $0.2-0.8$, depending on the sensor view angle and the Sun position.

\section{Land surface temperature}

Brightness temperatures (temperature of surface as "seen" by the satellite sensor) were extracted from the processed satellite scenes following the conventional algorithms for surface temperature retrieval (http://landsathandbook.gsfc. nasa.gov/handbook.html).

$T_{\mathrm{S}}=\frac{k_{2}}{\ln \left(\frac{\varepsilon \times k_{1}}{R_{\mathrm{C}}}+1\right)}$

Where $T_{\mathrm{S}}$ is the surface temperature $(\mathrm{K}) ; k_{1}$ and $k_{2}$ are calibration constants $\left(k_{1}=666.09 \mathrm{~W} / \mathrm{m}^{2} \mathrm{sr} \mu \mathrm{m} ; k_{2}=\right.$ $1,282,71 \mathrm{~K}) ; R_{\mathrm{C}}$ is the at-sensor radiance.

The thermal data were corrected for atmospheric effects. This step is essential in absolute temperature studies (Barsi et al. 2003). The Radiative Transfer Model available as a webbased tool (Barsi et al. 2003; http://atmcorr.gsfc.nasa.gov/) was used to estimate the transmission, upwelling, and downwelling radiance. The input data for the Radiative Transfer Model were acquired at the weather station at Malacky (Fig. 1). It would be outside the scope of this study to go into much detail concerning the temperature retrieval mechanism, and therefore, the reader is encouraged to consult appropriate literature covering this topic (e.g., Barsi et al. 2003; Sobrino and Caselles 1991; Sobrino et al. 1991; Qin et al. 2001).

\section{Results}

Raw satellite images were processed to obtain the NDVI and surface temperature applying Eq. 3 and Eq. 4, to be later used for the calculation of the TVDI according to Eq. 3. The unknown parameters $\left(a, b, T_{\mathrm{s} \_ \text {min }}\right)$ in Eq. 2 were identified iteratively in Solver MS Excel after setting the objective function (TVDI_max) equal to unity. The derived parameters " $a$ " and " $b$ " of Eq. 2 are listed in Table 1. Four Landsat ETM+ images were used to derive the TVDI index. Figure 6 shows the final product, i.e., maps of TVDI for the processed images. Note that the TVDI index exhibits a pronounced variability within a single scene (Fig. 6a, c). 
Fig. 6 Distribution of TVDI on a May 1, 2001; b May 14, 2000; c August 2, 2000; d August 24, 2002. For clarity, non-forest areas were masked out. TVDI levels are differentiated by color: red $(0.66<\mathrm{TVDI}<1.0)$; orange $(0.33<\mathrm{TVDI}<0.66)$; and pink $(0<\mathrm{TVDI} 0.33)$. The map scale is the same as in Fig. 1. Levels of fire risk for the individual scenes (calculated as Nesterov Index) are indicated in Table 2
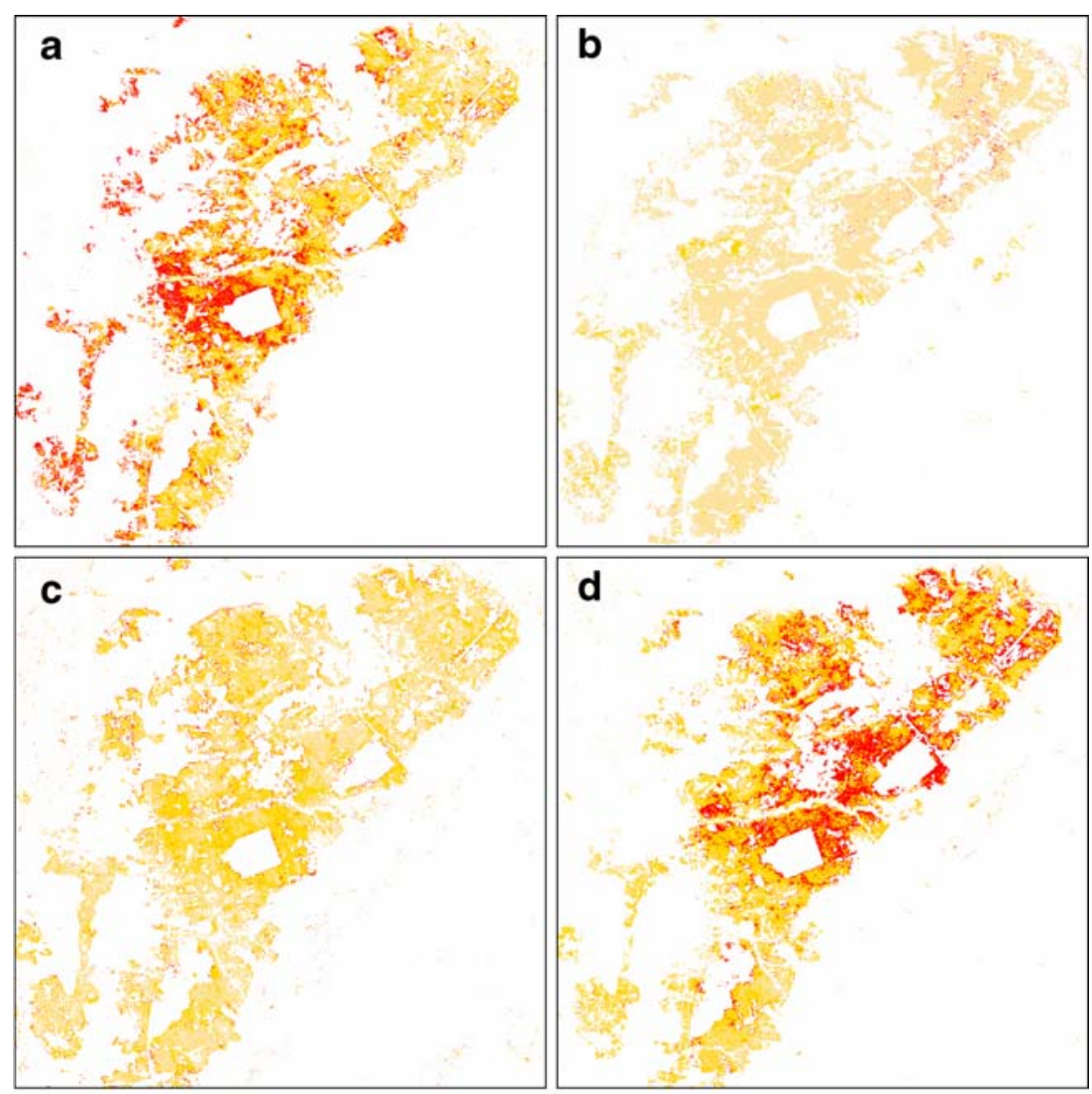

$<0.33$

$0.33-0.66$

$>0.66$
This variability suggests that the forest vegetation is not evenly moistened or is under different levels of fuel dryness. As shown in Table 2, NI may differ between two weather stations despite the relative short distance between them $(\sim 40 \mathrm{~km})$. This difference may be explained by the fact that precipitation is a highly variable phenomenon, i.e., there may be summer storm formation in one area, while a few kilometers away there is no rainfall. For example, on May 14, 2000, the NI calculated for the Malacky station (Fig. 1) was 5,156, while, at the Senica Station, the NI was as low as 1,224 . Therefore, relying merely on the NI derived from only one weather station may lead to misleading levels of fire risk, if the spatial character of the index is not taken into account. Using the Nesterov Index, or any other fire-rating index based on meteorological data, in synergy with the TVDI may be beneficial in terms of assigning each pixel in a satellite image an intrinsic fire-risk value. Figure 2 illustrates how traditional ground measurements of meteorological conditions can be merged with data from remote sensing. Note that the TVDI index can yield values only between zero and unity (Eq. 2). Dividing this interval into three subintervals $(0-0.33,0.33-0.66$, and 0.66-1.0) made it possible to roughly differentiate between pixels with "low" to "moderate" and "high" risk of fire outbreak. It should be noted that the choice to use only three intervals was rather arbitrary; certainly, one can divide the entire range of TVDI values into a finer subintervals. Calculating the Nesterov Index for the dates of the satellite image acquisition enabled us to assign the NI a spatial attribute when used with the spatially varying TVDI (Fig. 3). Sites with higher TVDI show where the risk of fire occurrence is more "likely" (Figs. 4 and 5). Sub-images of the area of interest (Fig. 6a-d) show the distribution of the TVDI on the dates of satellite overpass. On the other hand, the remaining TVDI maps (Fig. 6b-c) seem to be invariant with respect to the TVDI values; however, closer examination reveals that higher values of TVDI are present at forest edges. This is not surprising because experimental evidence exists that a canopy located at forest edges dries more quickly than the canopy within the forest owing to the enhanced evapotranspiration and decreased water storage near forest edges (Klaassen et al. 1996). Enhanced ventilation at the forest edges may also play a role. However, suspicion may arise in terms of incorrect interpretation of the forest edge effect, i.e., misclassification of mixed pixels. Generally, the higher the value of the 
TVDI the drier the conditions that are present within the analyzed pixels, hence, a higher chance of fire outbreak exists in areas where the TVDI exhibits elevated values. However, it should be noted here that TVDI values are independent of the Nesterov Index (or any other fire-risk rating index derived from weather data). Similar values of TVDI may be obtained on 2 days with different values of the Nesterov Index. In essence, TVDI serves as an "alert indicator" showing areas with a higher potential of fire outbreaks.

\section{Conclusions}

The presented technique may be of advantage especially in areas where weather stations are spread across long distances. It was shown that the Nesterov Index (Table 2) may substantially differ over short distances because precipitation is a highly variable phenomenon. In such instances, the calculated Nesterov Index (or any other weather-based index) may yield misleading results in terms of its spatial representativeness. The unknown perimeter within which a weather station accurately represents a studied area is the main limitation of all weather-based indices. Remotely sensed data may be used as supplementary attribute to traditional weather-based indices and serve as a useful tool for delineating sites with dry fuel conditions. We have shown that using traditional weather-based indices in synergy with satellite observations has important implications for fire management and prevention strategies in remote and vast forests with only limited fire-fighting resources and low coverage of meteorological stations.

Acknowledgment The authors wish to express their gratitude to the EU project GOCE 037063 "GEO-BENE" and MVTS (assessment of evapotranspiration and soil-vegetation dryness in forested areas of Slovakia by multispectral, thermal, and SAR satellite imagery) for providing necessary funding. The satellite images used in this paper were obtained from the USGS and the European Space Agency.

\section{References}

Barsi JA, Schott JR, Palluconi FD, Helder SJ, Hook SJ et al (2003) Landsat TM and ETM+ thermal band calibration. Can J Remote Sens 28:141-153

Bedient PB, Hubert WC, Vieux BE (2008) Hydrology and flood plain analysis. Forth edition. Pearson International Edition. PrenticeHall Inc. ISBN 13:978-0-13-242286-4, pp 29

Bowyer P, Danson FM (2004) Sensitivity of spectral reflectance to variation in live fuel moisture content at leaf and canopy level. Remote Sens Environ 92:297-308

Chuvieco E, Cocero D, Riano D, Martin P, Martinez-Vega J, de la Riva J, Perez F (2004) Combining NDVI and surface temperature for the estimation of fuel moisture content in forest fire rating. Remote Sens Environ 92:322-331
Danson FM, Bowyer P (2004) Estimating live fuel moisture content from remotely sensed reflectance. Remote Sens Environ 92:309-321

European Commission Report No. 5. Forest Fires in Europe 2004. S.P. I.05.147 EN, pp 30

Garcia-Martin A, Perez-Cabello F, de la Riva FJ, Lloveria RM (2008) Estimation of crown biomass of Pinus spp. From Landsat TM and its effect on burn severity in a Spanish fire scar. IEEE J Sel Top Appl Earth Observ 1:254-265

Goward SN, Cruickshanks GD, Hope AS (1985) Observed relation between thermal emission and reflected spectral radiance of a complex vegetated landscape. Remote Sens Environ 18:137-146

Hardy CC, Burgan RE (1999) Evaluation of NDVI for monitoring live fuel moisture in three vegetation types of the western U.S. Photogramm Eng Remote Sensing 65:603-610

Hernandez-Leal PA, Gonzalez-Calvo A, Arbelo M, Barreto A, Alonso-Benito A (2008) Synergy of GIS and remote sensing data in forest fire danger modeling. IEEE J Sel Top Appl Earth Observ 1(4):240-247

Klaassen W, Lankreijer HJM, Veen AWL (1996) Rainfall interception near a forest edge. J Hydrol 185:349-361

Nemani R, Pierce L, Running SN, Goward SN (1993) Developing satellite-derived estimates of surface moisture status. J Appl Meterol 32:548-557

Park S, Feddema JJ, Egbert SL (2004) Impacts of hydrologic soil properties on drought detection with MODIS thermal data. Remote Sens Environ 89:53-62

Peterson SH, Roberts DA, Dennison PE (2008) Mapping live fuel moisture with MODIS data: a multiple regression approach. Remote Sens Environ 112:4272-4284

Qin Z, Berliner P, Karnieli A (2001) A mono-window algorithm for retrieving land surface temperature from Landsat TM data and its application to Israel-Egypt border region. Int J Remote Sens 22:3719-3746

Richardson JJ, Moskal LM, Soo-Hyung K (2009) Modeling approaches to estimate effective leaf area index from aerial discrete-return LIDAR. Agric For Meteorol 149:1152-1160

Sandholt I, Rasmussen K, Andersen J (2002) A simple interpretation of the surface temperature/vegetation index space for assessment of surface moisture status. Remote Sens Environ 79:213-224

Shetinsky EA (1994) Protection of forests and forest pyrology. Ecology, Moscow (in Russian), p 209

Škvarenina J, Mindáš J, Holécy J, Tuček J (2004) An analysis of the meteorological conditions during two largest forest fire events in the Slovak Paradise National Park. Meteorological Journal 7:167-171

Sobrino JA, Caselles V (1991) A methodology for obtaining the crop temperature from NOAA-9 AVHRR data. Int J Remote Sens $12: 2461-2475$

Sobrino JA, Cull C, Caselles V (1991) Atmospheric correction for land surface temperature using NOAA-11 AVHRR channels 4 and 5. Remote Sens Environ 38:19-34

Tanskanen H, Venalainen A (2008) The relationship between fire activity and fire weather indices at different stages of the growing season in Finland. Boreal Environ Res 13:285-302

Tanskanen H, Venalainen A, Puttonen P, Granstrom A (2005) Impact of stand structure on surface fire ignition potential in Picea abies and Pinus sylvestris forests in southern Finland. Can J For Res $35: 410-420$

Tesař M, Šír M, Lichner L, Zelenková E (2006) Influence of vegetation cover on thermal regime of mountainous catchments. Biologia 61(Suppl. 19):311-314

Wang X, Xie H, Guan H, Zhou X (2007) Different responses of MODIS-derived NDVI to root-zone soil moisture in semi-arid and humid regions. J Hydrol 340:12-24

Xu D, Dai L, Shao G, Tang L, Wang H (2005) Forest fire risk zone mapping from satellite images and GIS for Baihe Forestry Bureau, Jilin, China. J For Res 16:169-174 